\title{
Depth Assessment using Contralateral Oblique View during Cervical InTERLAMINAR EPIDURAL STEROID INJECTIONS
}

\author{
Eric Helm, MD, and Michael O'Connell, DO
}

\begin{abstract}
Background: Recent literature has demonstrated that the contralateral oblique (CLO) view provides a more reliable angle in determining needle depth when compared to the lateral view during cervical interlaminar injections. Despite the utilization of CLO safety views, contrast patterns, and loss-of-resistance techniques, inherent risk of injury still remains. Additional safety measures must be assessed to provide clinicians with further safeguards to prevent procedural complications.

Objective: The purpose of our study is to provide a reliable method of gauging needle depth insertion during cervical interlaminar injections by comparing the distance from the skin to the epidural space when measured on magnetic resonance imaging (MRI) and when measured intraoperatively.

Methods: The study sample included 45 patients with cervical radiculopathy or cervical spinal stenosis. The distance from the skin to the epidural space was measured on cervical spine MRI and with the spinal needle intraoperatively. Primary
\end{abstract}

Cervical epidural steroid injections (CESI) are common procedures utilized in the treatment of cervical radiculopathy and cervical spinal stenosis. The use of fluoroscopic guidance is the standard of care. Traditional fluoroscopic views include the trajectory view (anterior-posterior) for initial needle placement

From : Department of Physical Medicine and Rehabilitation University of Pittsburgh Medical

Author for correspondence: Eric Helm, MD

Address: Department of Physical Medicine and Rehabilitation

University of Pittsburgh Medical

E-mail: helmer@upmc.edu analysis included the correlation between these distances, and whether differences in depth were influenced by injection level, needle tip location, or body mass index (BMI).

Results: A significant correlation $(r=.975, P<$ .001 ) with an average difference of $.03 \mathrm{~mm}$ (standard deviation, $2.99 \mathrm{~mm}$ ) was found between MRI and procedural measurements. Neither injection level nor BMI had a significant influence on the difference in depth.

Conclusion: When combined with traditional safety techniques, obtaining preprocedural MRI depth measurements can provide a reliable method in predicting the true needle depth to safely enter the epidural space. Because the majority of patients undergoing interlaminar cervical epidural steroid injections will have already obtained MRI, measuring the distance preprocedurally is a simple and practical method for physicians to implement.

Key words: Cervical, fluoroscopy, radiculopathy, injection, contra-lateral oblique, contrast, safety, complications, spinal stenosis, radiation, interlaminar and safety view (lateral) for needle depth. Anatomic visualization can be compromised in the lateral view, especially due to procedural neck positioning, obesity, short neck, and prior cervical spine surgery. The combination of suboptimal visualization, anatomic variability among patients, and the presence of the spinal cord increases the risk of significant procedural complications. For these reasons, utility of the contralateral-oblique (CLO) view for interlaminar CESI has recently gained more attention (1). The CLO view has been shown to provide improved needle depth assessment and visualization during interlaminar CESI (1-3). A multicenter survey of attitudes and perceptions of pain medicine fellows regarding the use of the lateral versus CLO view for interlaminar 


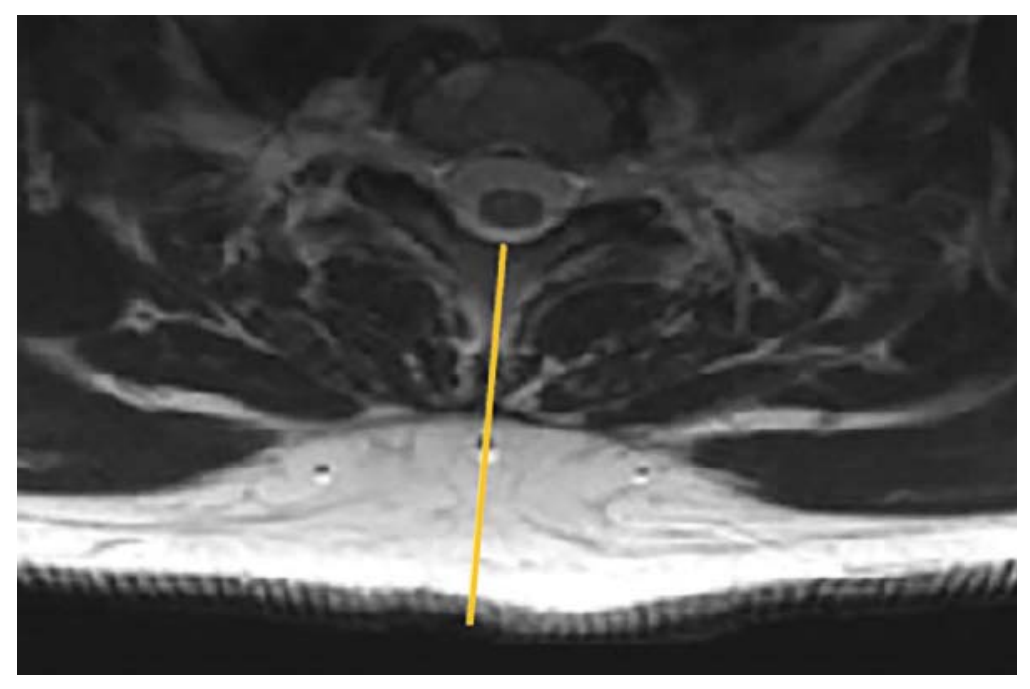

Fig. 1. Cervical spine magnetic resonance imaging (MRI) in axial view. This image was obtained for preprocedural planning, and the distance from the skin to the posterior epidural space was measured (indicated by the yellow arrow).

CESI indicated that the CLO technique was associated with improved anatomic assessments, superior visualization of contrast dye patterns, improved ease of learning, and lower perceived likelihood of complications (4).

Minimizing the amount of time that the fluoroscopic unit is on is the most important factor in keeping radiation doses as low as possible. Physicians utilize pulsed fluoroscopy, collimation, and distance from the radiation source as additional techniques to minimize radiation doses (5). The lateral and CLO safety views increase radiation exposure to the patient, but they are necessary to analyze needle depth. Independently viewing the pertinent anatomy preprocedurally is beneficial not only for maximizing therapeutic benefit and preventing complications, but also for increasing efficiency of the procedure and ultimately reducing radiation exposure to the patient.

In our study, we analyzed the correlation between the measured preprocedural cervical spine magnetic resonance imaging (MRI) needle depth with interlaminar CESI intraoperative needle depth. Our primary aim was to see how closely the depth assessment correlated. Accurate correlations may allow physicians performing interlaminar CESI the ability to decrease overall procedure time and minimize radiation dosage, reduce patient discomfort, and provide an additional safety measure in preventing complications such as dural puncture. Our secondary aim was to see if this correlation was influenced by either injection level (e.g., C6-7 or C7-T1) or needle tip location (e.g., midline vs off midline).

\section{METHODS}

We conducted a retrospective chart review of patients who underwent interlaminar CESI from January 2016 through October 2017. All procedures were performed in the Department of Physical Medicine and Rehabilitation at the University of Pittsburgh Medical Center. A cervical spine MRI was viewed for preprocedural planning. The measured distance from the skin to the targeted posterior epidural space was recorded in the patient's chart. The measurement was taken on a T2-weighted axial view utilizing the ruler function (Fig. 1). An interlaminar CESI was then performed with the typical standard of care. We utilized fluoroscopic imaging with anteroposterior (AP) and CLO views for needle visualization with every stage of advancing the needle. A 20-gauge epidural Tuohy needle was advanced from the skin surface to the ligamentum flavum, and a loss-of-resistance technique was utilized to access the posterior epidural space (Fig. 2). We then injected contrast dye through sterile extension tubing in the CLO view to confirm epidural spread and no intravascular or intradural placement. Once epidural flow was confirmed on multiple fluoroscopic views, a sterile blue ink marker was used to place a blue mark on the Tuohy needle at the skin surface. At this point, the medication 


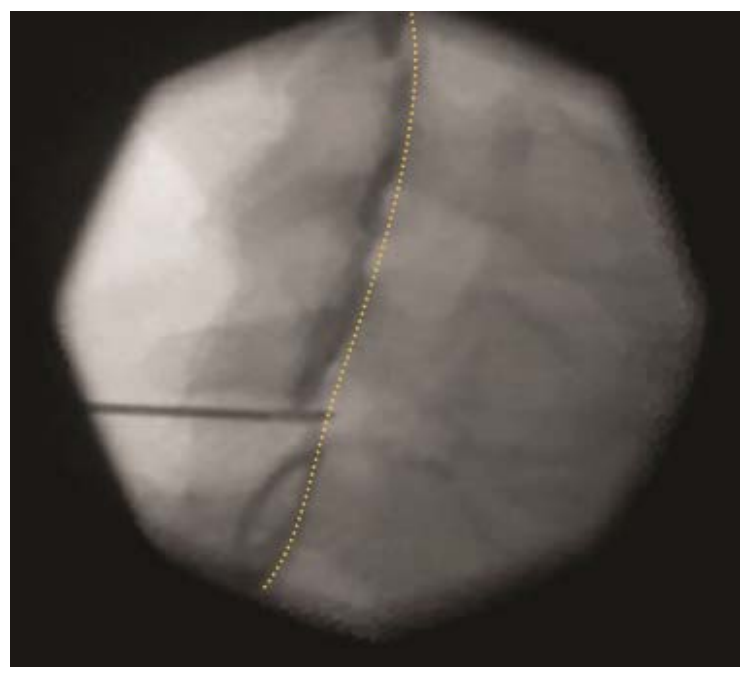

Fig. 2. Cervical spine radiograph, contralateral oblique view. This image was obtained to verify needle placement in the posterior epidural space (yellow line indicates spinolaminar line). A sterile skin mark was placed on the needle at the skin surface after confirming epidural placement with contrast dye. After needle removal, the distance from the needle tip to the intraoperative marking was recorded.

was injected, typically $10 \mathrm{mg}$ of dexamethasone 10 $\mathrm{mg} / \mathrm{mL}$ with $1 \mathrm{~mL}$ of $0.9 \%$ preservative-free normal saline. The Tuohy needle was removed and a sterile adhesive bandage was placed at the operative site. The actual procedural depth was then measured from the needle tip to the intraoperative marking. This measurement was recorded in the operative note.

\section{RESULTS}

The study sample included 45 patients with clinically diagnosed cervical radiculopathy, cervical radiculitis, or cervical spinal stenosis. Demographic data is shown in Fig. 3. The age range was from 19 to 82 years. The mean age was 55 years with a standard deviation (SD) of 14.4. The highest frequency of cases was in patients between 59 and 60 years old. The average body mass index (BMI) was 30.23 with a SD of 5.52. The most common level targeted was the $\mathrm{C} 7-\mathrm{T} 1$ vertebral interspace in 34 patients $(75.6 \%)$, and $88.9 \%$ were placed off midline. On average, the cervical spine MRI depth measurement was 68.23

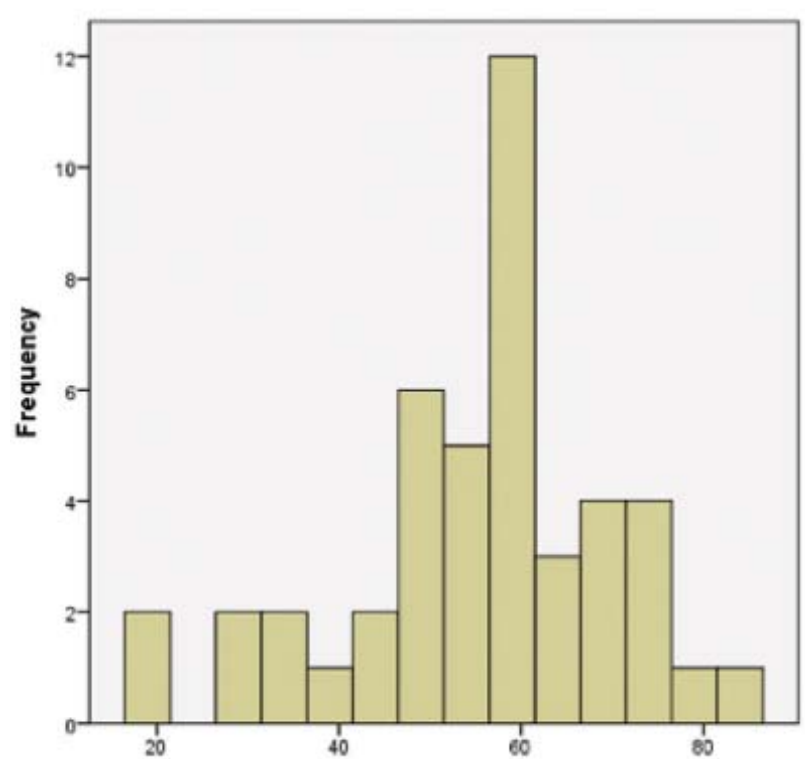

Fig. 3: Histogram detailing number of interlaminar cervical epidural steroid injections (CESI) per age group

$\mathrm{mm}$ with a SD of 12.22. The average procedure depth was very close to the average cervical spine MRI depth at $68.27 \mathrm{~mm}$ with a SD of 10.68 . There was a strong positive correlation between the MRI depth and actual procedural depth (Fig. 4). The Pearson correlation was $r=.975(P<.001)$. The average difference was small (mean $=-0.03 \mathrm{~mm}$ ) but variability in the sample led to a SD of 2.99. The largest discrepancy between MRI and procedure depth was $10 \mathrm{~mm}$, seen in 2 cases (Fig. 5). Using a 2-tailed an independent samples $t$ test, the mean difference between the $\mathrm{MRI}$ and procedure depth was not significantly different for the C6-7 group (mean $=-0.68, \mathrm{SD}=2.8$ ) when compared to the C7-T1 group (mean $=0.29$, $\mathrm{SD}=3.2) ; \mathrm{t}=-0.86, P=.395$. There was no visible relationship between $\mathrm{BMI}$ and differences in $\mathrm{MRI}$ and procedural depth (Pearson correlation of $r=0.178 ; P$ $=.243)$. The needle tip location analysis was unable to be performed due to an unbalanced sample size of the midline group $(n=5)$ versus left/right $(n=40)$.

\section{DISCUSSION}

Interlaminar CESI are often utilized in the treatment of radicular pain. However, due to the close proximity of vital structures within the cervical spine, inaccurate 


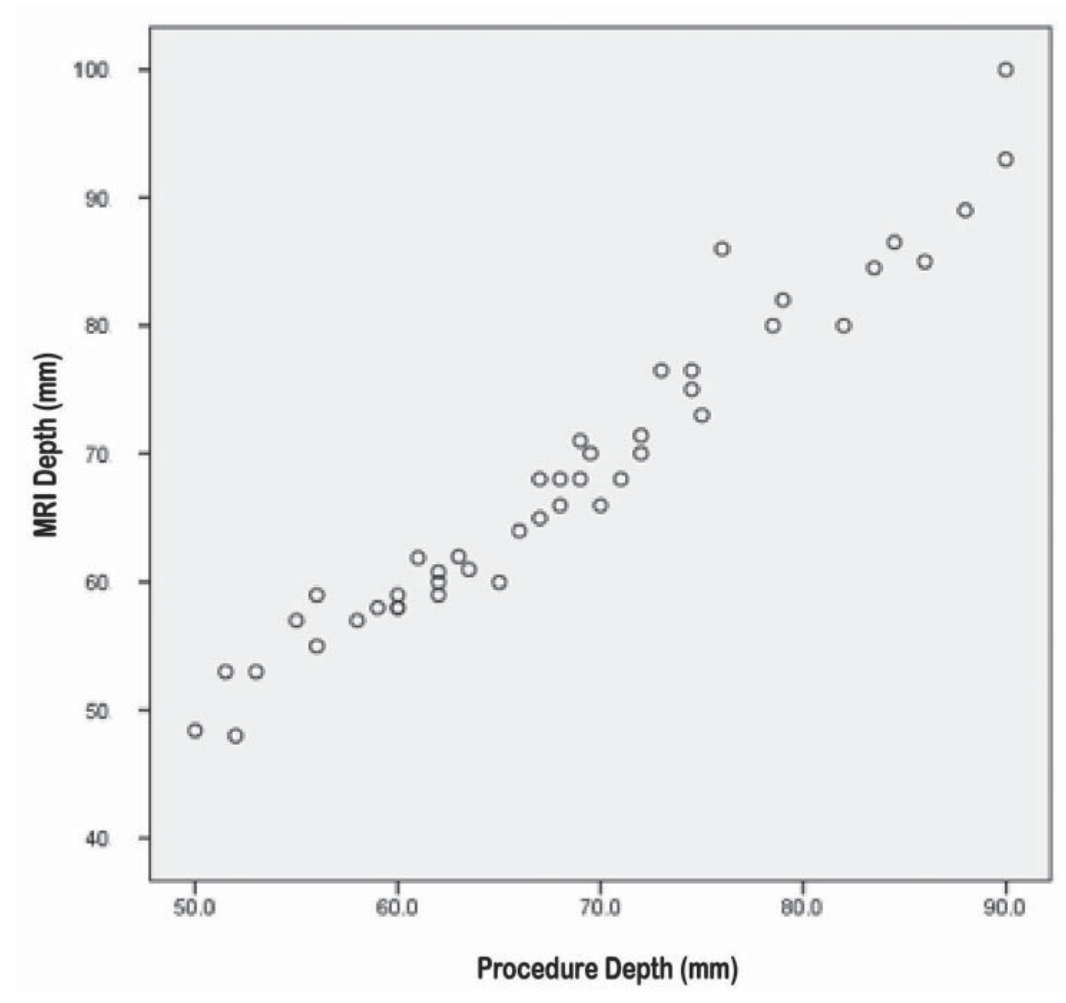

Fig. 4. Correlation between MRI depth and intraoperative depth performance could lead to severe complications, including dural puncture and spinal cord injury (6). In addition, the high prevalence of anatomic variability of the ligamentum flavum at the cervical level further increases the risk of encountering false loss of resistance (LOR) (7). Despite implementation of various safety methods, including utilization of the CLO view for accurate needle depth assessment and needle advancement with concurrent use of contrast medium, inherent risk of injury remains $(1,6)$.

Our study has shown a strong correlation between preprocedural cervical spine MRI and intraoperative depth measurements. In the majority of cases, our study demonstrated that obtaining preprocedural MRI depth measurements can provide a reliable method in predicting the true needle depth to safely enter the posterior epidural space. Despite these results, our findings should be interpreted with caution. There was a standard deviation of 2.99 between MRI and procedural measurements and 2 cases had discrepancies of $10 \mathrm{~mm}$. If one was to rely solely on axial MRI measurements to gauge needle depth, this would likely lead to inaccurate placement and ultimately procedural complications. However, when combined with traditional safety techniques, reviewing axial MRI measurements can provide a reasonably accurate estimate of the distance to the posterior epidural space in the majority of cases. Previous literature has advocated for this technique, as this can identify for the presence of ligamentum flavum hypertrophy and aid in selecting the appropriate angle of entry (8).

Utilizing this method may also increase the overall efficiency of the procedure by minimizing the usage of safety views, which in turn decreases fluoroscopic time and minimizes radiation exposure to the patient. Because the majority of patients undergoing interlaminar CESI will have already obtained MRI, measuring the distance preprocedurally is a simple and practical method for physicians to implement.

This study has several limitations. Our ability to analyze secondary outcome measures, which explored whether depth correlation was influenced by either injection level or needle tip location, was limited due 
Fig. 5. On the x-axis, the positive values represent MRI measurements that were greater than intraoperative measurements, while the negative values represent intraoperative measurements that were greater than MRI measurements. The y-axis represents number of cases.

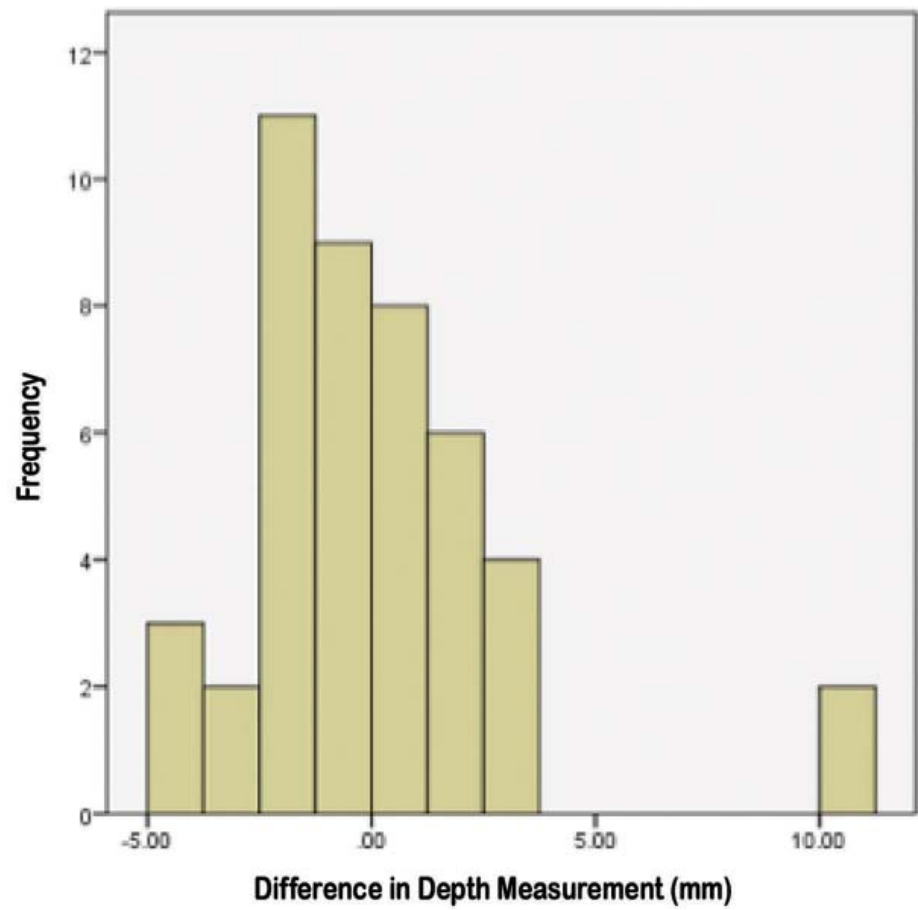

to small sample size; we were unable to draw statistically significant information from these variables. It is possible that a larger sample size could elucidate a difference among these factors, and this question warrants further study.

Additionally, all procedures and measurements were performed by the same interventionalist, and blinding did not occur. Finally, the study was retrospective and there was no control group or randomization. Future research investigating whether obtaining preprocedural MRI measurements improves pain scores, functional outcomes, and complication rates in patients undergoing interlaminar CESI could potentially yield valuable information.

\section{CONCLUSION}

This retrospective study showed a strong correlation between preprocedural cervical spine MRI and intraoperative depth measurements. When utilizing the CLO view during interlaminar CESI, obtaining preprocedural MRI depth measurements can provide a reliable method in predicting needle depth insertion to the epidural space, improving the safety and efficiency of this procedure. 


\section{REFERENCES}

1. Landers $M H$, Dreyfuss $P$, Bogduk N. On the geometry of fluoroscopy views for cervical interlaminar epidural injections. Pain Med 2012; 13:58-65.

2. Furman MB, Jasper NR, Lin HW. Fluoroscopic contralateral oblique view in interlaminar interventions: A technical note. Pain Med 2012 Nov; 13:1389-1396.

3. Gill JS, Aner M, Nagda JV, Keel JC, Simopoulos TT. Contralateral oblique view is superior to lateral view for interlaminar cervical and cervicothoracic epidural access. Pain Med 2015; 16:68-80.

4. Jain G, Helm ER, Nedeljkovic SS, Wasan AD, Wang H. Multicenter survey of attitudes and perceptions of pain medicine fellows toward the use of lateral versus contralateral-oblique fluoroscopic view for interlaminar cervical epidural injection. Pain Med 2015; 16:692-695.
5. Goodman BS, Carnel CT, Mallempati S, Agarwal P. Reduction in average fluoroscopic exposure times for interventional spinal procedures through the use of pulsed and low-dose image settings. Am J Phys Med Rehabil 2011; 90:908-912.

6. Abbasi A, Malhotra G, Malanga G, Elovic EP, Kahn S. Complications of interlaminar cervical epidural steroid injections: A review of the literature. Spine 2007; 32:2144-2151.

7. Lirk P, Kolbitsch C, Putz G, Colvin J, Colvin HP, Lorenz I, Keller C, Kirchmair L, Rieder J, Moriggl B. Cervical and high thoracic ligamentum flavum frequently fails to fuse in the midline. Anesthesiology 2003; 99:1387-1390.

8. Gill JS, Aner M, Simopoulos TT. Intricacies of the contralateral oblique view for interlaminar epidural access. Pain Med 2013; $14: 1265-1268$. 